\title{
Health considerations of the legalization of cannabis edibles
}

\author{
Jasleen K. Grewal MD, Lawrence C. Loh MD MPH
}

Cite as: CMAJ 2020 January 6;192:E1-2. doi: 10.1503/cmaj.191217

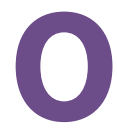

n Oct. 17, 2019, Canada's amended cannabis regulations entered into force, authorizing the legal production and sale of cannabis edibles in the next phase of the country's legalization journey. Edible products are anticipated to become publicly available after the required 60-day notice period for federal licence holders to advise Health Canada of their intent to sell new products. These products are already popular, as evidenced by the 2019 National Cannabis Survey, which found that $27 \%$ of respondents who had used cannabis in the past 3 months had consumed edible forms, presumably through home preparation or from illegal channels..$^{1,2}$ Although edibles are commonly viewed as a safer and more desirable alternative to smoked or vaped cannabis, physicians and the public should be aware of several risks related to the use of cannabis edibles.

Foremost among these is the association of cannabis edibles with a longer latency and duration of effects than inhaled cannabis, which may increase the risk of overdosing from overconsumption. The onset of psychoactive effects from cannabis edibles can be delayed by up to 4 hours after consumption, and the effects can last for more than 8 hours overall, which lengthens the duration of impaired judgment and coordination experienced in comparison to inhaled cannabis. ${ }^{3}$ Previously, variability in dosing information also increased the risk of overconsumption. To address this, the new regulations mandate the use of standard dosing information (i.e., milligrams of tetrahydrocannabinol [THC] per unit or serving) on cannabis edibles for purchase and restricts edible composition to a limit of $10 \mathrm{mg}$ of THC per edible unit or serving. Despite this, even while following dosing recommendations, individuals' responses to different products may vary and overdosing may still occur, with cannabisnaive individuals particularly at risk. ${ }^{4}$

Two populations at high risk of harm from cannabis edibles are children and older adults. Children (and pets) are at risk of accidental ingestion and overconsumption of cannabis because many edibles resemble candy or other food and drink. After legalization of cannabis edibles in Colorado, the state poison control centre saw a $70 \%$ increase in calls for accidental cannabis exposure in children from 2013 to 2017, and studies of health care usage reported more children than adults being treated for ingestion

\section{KEY POINTS}

- Recent regulatory changes allow Canadians legal access to cannabis edibles, which are already frequently consumed among those who use cannabis.

- Amended regulations concerning edibles limit dosing, prohibit formulations with other psychoactive substances, and require safety measures with regard to packaging and production.

- Cannabis edibles present specific risks of overconsumption and accidental ingestion, especially among cannabis-naive individuals and children, and additional risks for youth and senior populations with regard to mental well-being and cognitive functioning.

- Physicians should openly discuss patients' use of cannabis, including edibles, to enable counselling on safe consumption and driving, and to discourage the consumption of illicit or homemade edibles.

- Continuing surveillance and advocacy efforts are needed to identify and address unforeseen effects on communities.

incidents. ${ }^{3}$ People aged 65 years and older report the lowest rate of cannabis use overall, but use in this group has reportedly increased after legalization in Canada, mirroring a trend in the United States in which older adults increasingly report cannabis use to manage symptoms of chronic conditions amid changing social norms. ${ }^{2,5}$ Among older adults, cannabis consumption including use of edibles - has been linked to greater cognitive impairment and a heightened risk of hypotension-related falls, arrhythmia and drug interactions. ${ }^{6}$

Youth represent another population of concern, and a survey from the World Health Organization found that 15-year-old Canadians reported lifetime and past-month cannabis use at a higher rate than peers in other high-income countries. ${ }^{7} \mathrm{~A}$ recent Canadian report highlighted a pervasive belief among youth that consuming cannabis edibles has a positive effect on mood, anxiety and sleep. ${ }^{8}$ This perception is at odds with described harms related to cannabis use among youth, particularly impaired brain development and poor mental health associated with initiation in early adolescence. ${ }^{7}$ Finally, longer-term use of edibles has also been associated with increased risks of adverse effects, such as panic attacks, psychosis and hyperemesis syndrome. ${ }^{1}$ 
Using the 2016 recommendations of the Canadian Task Force on Cannabis Legalization and Regulation as a guide, the amended regulation that legalizes edibles aims to address potential health risks. In addition to the dosing limits described above, there are also restrictions on the use of psychoactive ingredients with which edible cannabis can be combined, to guard against dependence. Formulations must not contain nicotine and alcohol, and can contain only a limited amount of caffeine. $^{9}$

Canadian regulations also require edibles to be stored in plain, child-resistant packaging to prevent accidental ingestion, and require a standardized symbol and health warning with limits on various marketing and promotional claims. Strict manufacturing controls and a prohibition on producing food and cannabis in the same facility are also required, probably to ensure product consistency while minimizing cross-contamination or mix-up with conventional foodstuffs. ${ }^{9}$

Physicians should routinely question patients who ask about cannabis about their use or intended use of edible cannabis products so that they can counsel these patients regarding child safety, potential for accidental overconsumption and delayed effects, and potential for interactions with other substances such as alcohol, benzodiazepines, sleeping aids and opioids. Physicians should advise strongly against driving after consuming cannabis edibles, particularly if other drugs have been used.

Physicians should also discourage the consumption of illicit or homemade cannabis edibles. Risks of such products include spread of foodborne illness, overdose owing to variable THC content of products, poisoning from pesticide residues, and potential for unexpected effects given that illicit products may be contaminated with other drugs, such as narcotics. ${ }^{10}$

Physicians, patients and communities must work together to keep health and safety considerations at the forefront of ongoing review and regulation of the production, sale and consumption of edible cannabis products in Canada. Under amended legislation, cannabis edibles cannot be legally sold to youth. Evidence suggests that interactive school-based education programs are associated with reduced cannabis use among youth compared with didactic programs. ${ }^{11}$ As little is known about the effects of cannabis use in older adults, researchers should aim to identify relevant risk factors and interventions for this group.

All populations will benefit from ongoing monitoring and surveillance to evaluate the effects of legalized cannabis edibles. Continued advocacy for effective regulation of edibles will help to control the availability of both legal and illicit products and mitigate their effects on individual and community health.

\section{References}

1. Barrus DG, Capogrossi KL, Cates SC, et al. Tasty THC: promises and challenges of cannabis edibles. Methods report (RTI Press) 2016 Nov.;2016. doi: 10.3768/ rtipress.2016.op.0035.1611.

2. National Cannabis Survey - Table 5. Number and percentage reporting consumption of cannabis in the past three months by product and gender, household population aged 15 years or older, Canada, first half of 2019. Ottawa: Statistics Canada; modified 2019 Aug. 15. Available: www150.statcan.gc.ca/n1/ daily-quotidien/190815/t005a-eng.htm (accessed 2019 Oct. 29).

3. Ghosh TS, Van Dyke M, Maffey A, et al. Medical marijuana's public health lessons implications for retail marijuana in Colorado. N Engl J Med 2015;372:991-3.

4. Hammond D. Communicating THC levels and 'dose' to consumers: implications for product labelling and packaging of cannabis products in regulated markets. Int J Drug Policy 2019 Jul 24. pii: S0955-3959(19)30182-3. doi: 10.1016/j.drugpo.2019.07.004. [Epub ahead of print].

5. Kaskie B, Ayyagari P, Milavetz G, et al. The increasing use of cannabis among older Americans: A public health crisis or viable policy alternative? Gerontologist 2017;57:1166-72.

6. van den Elsen GA, Ahmed Al, Lammers M, et al. Efficacy and safety of medical cannabinoids in older subjects: a systematic review. Ageing Res Rev 2014;14:56-64.

7. Inchley J, Currie D, Young T, et al., editors. Growing up unequal: gender and socioeconomic differences in young people's health and well-being. Copenhagen: World Health Organization - European Regional Office; 2016.

8. Canadian youth perceptions on cannabis. Ottawa: Canadian Centre on Substance Abuse and Addiction; 2017. Available: www.ccsa.ca/canadian-youth -perceptions-cannabis-report (accessed 2019 Oct. 30).

9. Final regulations: edible cannabis, cannabis extracts, cannabis topicals. Ottawa: Health Canada; 2019. Available: www.canada.ca/en/health-canada/ services/drugs-medication/cannabis/resources/regulations-edible-cannabis -extracts-topicals.html (accessed 2019 Oct. 30).

10. Special concerns associated with cannabis extractions, concentrations, infusions, and infused foods. Denver: Department of Public Health and Environment; 2019. Available: www.denvergov.org/content/dam/denvergov/Portals/771/documents/ $\mathrm{PHI} /$ Food/SpecialConcerns\%20withCannabisExtractions.pdf (accessed 2018 Dec. 26).

11. Porath-Waller AJ, Beasley E, Beirness DJ. A meta-analytic review of schoolbased prevention for cannabis use. Health Educ Behav 2010;37:709-23.

\section{Competing interests: None declared.}

This article has been peer reviewed.

Affiliations: Department of Family and Community Medicine (Grewal), University of Toronto, Toronto, Ont.; Public Health, Region of Peel (Loh), Mississauga, Ont.; Dalla Lana School of Public Health (Loh), University of Toronto, Toronto, Ont.

Contributors: Both authors contributed equally to the conception, drafting and revision of the work, approved the final version to be published, and agreed to be accountable for all aspects of the work.

Correspondence to: Lawrence Loh, lawrence.loh@peelregion.ca 